\title{
Affine generalizations of gravity in the light of modern cosmology
}

\author{
A.T. Filippov * \\ + Joint Institute for Nuclear Research, Dubna, Moscow Region RU-141980
}

September 28, 2018

\begin{abstract}
We discuss new models of an 'affine' theory of gravity in multidimensional space-times with symmetric connections. We use and develop ideas of Weyl, Eddington, and Einstein, in particular, Einstein's proposal to specify the space - time geometry by use of the Hamilton principle. More specifically, the connection coefficients are determined using a 'geometric' Lagrangian that is an arbitrary function of the generalized (non-symmetric) Ricci curvature tensor (and, possibly, of other fundamental tensors) expressed in terms of the connection coefficients regarded as independent variables. Such a theory supplements the standard Einstein gravity with dark energy (the cosmological constant, in the first approximation), a neutral massive (or tachyonic) vector field (vecton), and massive (or tachyonic) scalar fields. These fields couple only to gravity and can generate dark matter and/or inflation. The new field masses (real or imaginary) have a geometric origin and must appear in any concrete model. The concrete choice of the geometric Lagrangian determines further details of the theory, for example, the nature of the vector and scalar fields that can describe massive particles, tachyons, or even 'phantoms'. In 'natural' geometric theories, which are discussed here, dark energy must also arise. We mainly focus on intricate relations between geometry and dynamics while only very briefly considering approximate cosmological models inspired by the geometric approach.
\end{abstract}

\section{Introduction}

Attempts to modify Einstein's general relativity began immediately after the general relativity was formulated in its final form (1915 -1916). Einstein himself added the cosmological constant term $\Lambda$ to save his static cosmology. After Friedmann's work (1922-1924) this modification was becoming more and more dubious. In 1918, Weyl developed a much more serious modification aimed at 'unifying' gravity and electromagnetism (it is most clearly summarized in [1]). Starting from LevyCivita's ideas on non-Riemannian connections (1917), he developed a theory of a manifold equipped with the connection that depends both on a metric tensor and on a vector field, which he attempted to identify with the electromagnetic potential. To get a consistent theory, Weyl introduced a general idea of gauge invariance which survived although the theory itself failed as he admitted later. At the same time, he invented conformal transformations and the conformal curvature tensor (Weyl's tensor).

In 1919, Eddington proposed a more radical modification of general relativity [2], 3]. His idea was to start with a pure affine formulation of the gravitation, i.e. to first use the general symmetric affine connection and only at some later stage to introduce a metric tensor. Eddington proposed

\footnotetext{
*Alexandre.Filippov@jinr.ru
} 
several interesting ideas, including Eddington's density discussed below, but he could not construct a logically consistent theory based on an affine connection.

Einstein, starting from Eddington's ideas developed a consistent theory based on using the Hamilton principle. His theory was presented in three beautiful and concise papers [4], later summarized by Eddington [2] and Einstein [5] and soon forgotten (but see brief discussions in [6], [7]). The Einstein models were recently reinterpreted in [8] and [9], where a brief summary of the ideas and results in those papers that are of interest for modern investigations can be found.

Einstein's key idea was to derive the concrete form of the affine connection by applying the Hamilton principle to a generic Lagrangian depending on the generalized Ricci curvature. This assumption completely fixes a geometry, which does not coincide with Weyl's geometry, but belongs to the same simple class recently introduced and discussed in [8]. Einstein's unusual result was difficult to comprehend in twentieth of the last century and it remains somewhat puzzling these days. From the modern mathematics viewpoint, its origin could be ascribed to a sort of a mismatch between the affine connection geometry and the Lagrangian 'geometry'. At the moment, it is difficult to find a more detailed explanation. Possibly, this is an interesting mathematical problem.

A more detailed presentation of the main ideas and results discussed here can be found in $[8]$ and [9], but we warn the reader that there are several essential changes in our approach to some problems. In Section 2 we give a very brief summary of the affine connection theory. In Section 3 we expose Einstein's approach, formulate general geometric and physical requirements to the theory and their simplest realization. In Section 4 we discuss simple cosmological models.

\section{Geometry}

Weyl's basic idea was that unifying gravity and electromagnetism requires using a non-Riemannian symmetric connection. In general, the connection coefficients can be expressed in terms of the Riemannian connection $\Gamma_{j k}^{i}$ and of an arbitrary third rank tensor $a_{j k}^{i}$ that is symmetric in the lower indices

$$
\gamma_{j k}^{i}=\Gamma_{j k}^{i}[g]+a_{j k}^{i}
$$

Here $g_{i j}$ is an arbitrary symmetric tensor and $\Gamma_{j k}^{i}[g]$ is its Christoffel symbol

$$
\Gamma_{j k}^{i}[g]=\frac{1}{2} g^{i l}\left(g_{l j, k}+g_{l k, j}-g_{j k, l}\right)
$$

where the commas denote differentiations and $g_{i j} g^{j k}=\delta_{i}^{k}$. More precisely, for any symmetric connection, there exists a symmetric tensor $g_{i j}$ and a tensor $a_{j k}^{i}=a_{k j}^{i}$ such that (1) is satisfied.

The curvature tensor can be defined without using any metric:

$$
r_{j k l}^{i}=-\gamma_{j k, l}^{i}+\gamma_{m k}^{i} \gamma_{j l}^{m}+\gamma_{j l, k}^{i}-\gamma_{m l}^{i} \gamma_{j k}^{m}
$$

Then, the Ricci-like (but non-symmetric) curvature tensor can be defined by contracting the indices $i, l$ (or, equivalently, $i, k$ ):

$$
r_{j k}=-\gamma_{j k, i}^{i}+\gamma_{m k}^{i} \gamma_{j i}^{m}+\gamma_{j i, k}^{i}-\gamma_{m i}^{i} \gamma_{j k}^{m}
$$

(we again stress that $\gamma_{j k}^{i}=\gamma_{k j}^{i}$ but $r_{j k} \neq r_{k j}$ ). Using only these tensors and the antisymmetric tensor density, we can construct a quite rich geometric structure 1

The symmetric part of the Ricci curvature $r_{i j}$,

$$
s_{i j} \equiv \frac{1}{2}\left(r_{i j}+r_{j i}\right)
$$

\footnotetext{
${ }^{1}$ The geometry of symmetric and nonsymmetric connections is carefully reviewed in [10] and in [6].
} 
and its antisymmetric part,

$$
a_{i j} \equiv \frac{1}{2}\left(r_{i j}-r_{j i}\right)=\frac{1}{2}\left(\gamma_{j m, i}^{m}-\gamma_{i m, j}^{m}\right), \quad a_{i j, k}+a_{j k, i}+a_{k i, j} \equiv 0,
$$

have essentially different roles in geometry and in physics. The antisymmetric tensor $a_{i j}$ strongly resembles the electromagnetic field tensor and is actually related to the massive (or tachyonic) vector field (vecton), which is proportional to the vector $a_{i} \equiv a_{i m}^{m}$ (or to $\gamma_{i} \equiv \gamma_{i m}^{m}$ ). According to (1),

$$
a_{i} \equiv \gamma_{m i}^{m}-\Gamma_{m i}^{m} \equiv \gamma_{i}-\partial_{i} \ln \sqrt{|g|},
$$

where $g \equiv \operatorname{det}\left(g_{i j}\right)$. Therefore the vectors $a_{i}$ and $\gamma_{i} \equiv \gamma_{i m}^{m}$ differ by a gauge transformation, which played and important role in Weyl's theory, but it is not so important for us, at the moment. It is important that $a_{i j}$ can be simply expressed in terms of the vector $a_{i}$ (or $\gamma_{i}$ )

$$
a_{i j} \equiv-\frac{1}{2}\left(a_{i, j}-a_{j, i}\right) \equiv-\frac{1}{2}\left(\gamma_{i, j}-\gamma_{j, i}\right),
$$

Eddington's scalar density

$$
\mathcal{L} \equiv \sqrt{-\operatorname{det}\left(r_{i j}\right)} \equiv \sqrt{-r},
$$

which resembles the fundamental scalar density of the Riemannian geometry, $\sqrt{-\operatorname{det}\left(g_{i j}\right)} \equiv \sqrt{-g}$, is also an important geometric and physical object. Einstein used it as the Lagrangian in his first paper on affine model. Here, we discuss more general Lagrangians that can be obtained using densities constructed of $s_{i j}, a_{i j}$, and $a_{k}$.

Introducing the covariant derivative $\nabla_{i}^{\gamma}$ (with respect to the connection $\gamma$ ) we can rewrite the symmetric part of the curvature as

$$
s_{i j}=-\nabla_{m}^{\gamma} \gamma_{i j}^{m}+\frac{1}{2}\left(\nabla_{i}^{\gamma} \gamma_{j}+\nabla_{j}^{\gamma} \gamma_{i}\right)-\gamma_{n i}^{m} \gamma_{m j}^{n}+\gamma_{i j}^{n} \gamma_{n}
$$

Using the 'metric' covariant derivative $\nabla_{i}^{g} \equiv \nabla_{i}$ we can rewrite $s_{i j}$ in the form

$$
s_{i j}=R_{i j}[g]-\nabla_{m} a_{i j}^{m}+\frac{1}{2}\left(\nabla_{i} a_{j}+\nabla_{j} a_{i}\right)+a_{n i}^{m} a_{m j}^{n}-a_{i j}^{m} a_{m},
$$

where $R_{i j}[g]$ is the standard Ricci tensor of a Riemannian space with the metric $g_{i j}$.

For a general symmetric connection one can introduce the concept of the geodesic curve, the tangent vector to which is parallel to itself at every point of the curve. Eisenhart [10] calls these curves 'paths', but we may also call them geodesic curves (or geodesics) because they directly generalize the geodesics of the Riemannian geometry. The equations for geodesic curves of any symmetric connection $\gamma_{j k}^{i}$ can be written in the form

$$
\ddot{x}^{i}+\gamma_{j k}^{i} \dot{x}^{j} \dot{x}^{k}=0,
$$

where the dot denotes differentiating with respect to the so called 'affine' parameter $\tau$ of the curve $x^{i}(\tau)$. Using the affine parameter we can compare the distances between points on the same curve.

For a particular path, the affine parameter is unique up to an affine transformation $\tau \mapsto \tau^{\prime}=$ $a \tau+b$. Each connection define the unique set of paths, but all symmetric connections

$$
\hat{\gamma}_{j k}^{i}=\gamma_{j k}^{i}+\delta_{j}^{i} \hat{a}_{k}+\delta_{k}^{i} \hat{a}_{j},
$$

with an arbitrary vector $\hat{a}_{k}$, define the same paths. The Weyl (conformal) tensor $W_{j k l}^{i}$ of connection (12) is independent of $\hat{a}_{k}$ while the Ricci tensor and its symmetric and antisymmetric parts are $\hat{a}_{i}$-dependent (see [10] for more details). 
An interesting class of connections is

$$
\hat{\gamma}_{j k}^{i}=\Gamma_{j k}^{i}[g]+\delta_{j}^{i} \hat{a}_{k}+\delta_{k}^{i} \hat{a}_{j},
$$

where $\Gamma_{j k}^{i}[g]$ is a Riemannian connection (the Christoffel symbol of a symmetric tensor $g_{i j}$ ). The paths of the connection $\hat{\gamma}_{j k}^{i}$ coincide with the geodesics of $\Gamma_{j k}^{i}[g]$, but the Ricci tensor of $\hat{\gamma}$ is symmetric if and only if $\hat{a}_{i}=\partial_{i} \hat{a}$ with some scalar $\hat{a}$. We see that connection (13) is maximally close to the Riemannian connection $\Gamma_{j k}^{i}[g]$ and may be called a 'geodesically Riemannian' (or $g$ Riemannian) connection. Weyl and Einstein studied more general connections that belong to the following class introduced in [8], [9]:

$$
\gamma_{j k}^{i}=\Gamma_{j k}^{i}[g]+\alpha\left(\delta_{j}^{i} \hat{a}_{k}+\delta_{k}^{i} \hat{a}_{j}\right)-(\alpha-2 \beta) g_{j k} \hat{a}^{i},
$$

where $\hat{a}^{i} \equiv g^{i m} \hat{a}_{m}$. The Weyl connection corresponds to $\beta=0$ and the $g$-Riemannian connection, to $\alpha=2 \beta$. Einstein derived the connection for the space-time dimension $D=4$, his result is $\alpha=-\beta=\frac{1}{6}$ (we generalize it to any dimension in the next section).

Using (10) it is easy to calculate the physically important expression for the symmetric part of the Ricci curvature. The terms linear in $A$ are equal to

$$
(\alpha+\beta)\left(\nabla_{i} \hat{a}_{j}+\nabla_{j} \hat{a}_{i}\right)+(\alpha-2 \beta) g_{i j} \nabla_{m} \hat{a}^{m},
$$

and the quadratic terms are

$$
\hat{a}_{i} \hat{a}_{j}\left[(\alpha-2 \beta)^{2}-3 \alpha^{2}\right]+2 g_{i j} \hat{a}^{2}(\alpha-2 \beta)(\alpha+\beta) .
$$

It is easily seen that the sign of the first term in (16) can be positive or negative, but the second term in (16) and the linear terms in (10) are nonzero in the general case.

Before we leave pure mathematics and turn to more physical problems, we should mention one of the characteristic properties of symmetric connections. For applications of geometry to gravity, it is very important that at every point of the affine-connected space-time manifold there must exist a geodesic coordinate system, such that the connection coefficients are zero at this point. Using the above formulas it is easy to prove that such a coordinate system exists if and only if the connection is symmetric. For symmetric connections, the Fermi theorem about the existence of geodesic coordinates along the curves also holds (for the precise definitions and proofs see [10]).

\section{From Geometry to Dynamics}

Einstein's approach to constructing the generalized theory of gravity consists of two stages. In the first stage, he assumed that the general symmetric connection should be restricted by the Hamilton principle for a general Lagrangian density depending either on $r_{i j}$ (in the first two papers) or on $s_{i j}$ and $a_{i j}$ separately (in the third paper) 2 He gave no motivation for this assumption, but it is easy to see that the resulting theory in the limit $a_{i j}=0$ is consistent with the standard general relativity supplemented with a cosmological term. In this stage, Einstein succeeded in deriving the remarkable expression (14) for the connection (with $\alpha=-\beta=\frac{1}{6}$ ) and the general expression for $s_{i j}$ depending on a massive (tachyonic) vector field and the metric tensor density $\mathbf{g}^{i j}$.

\footnotetext{
${ }^{2}$ This idea was quite alien to Weyl and Eddington, who began by formulating a particular geometry. They therefore postulated the connection (14) with $\beta=0$ and then tried to write some equations generalizing the Einstein equations. Although the general approaches and the connection coefficients differed, the equations considered by the three authors have many features in common. In particular, the nonzero cosmological constant (exactly or in an approximation) and massive (tachyonic) vector field.
} 
In the next stage, a concrete Lagrangian density $\mathcal{L}\left(s_{i j}, a_{i j}\right)$ should be chosen. Einstein did not formulate any principle for selecting a Lagrangian, and both from geometric and physical standpoint his concrete choice seems sufficiently arbitrary, especially in the third paper. We believe that his best choice was made in the first two papers and, indeed, very similar effective Lagrangians are considered in modern applications of the superstring theory to cosmology.

Let us try to formulate requirements for a geometric Lagrangian density $\mathcal{L}$ :

1. It is independent of dimensional constants.

2. Its integral over the $D$-dimensional space-time is dimensionless.

3. It can depend on tensor variables having a direct geometric meaning and a natural physical interpretation.

4. Most importantly, the resulting generalized theory must agree with the well-established experimental consequences of the standard Einstein theory.

The last requirement is rather difficult to check without a detailed development of the theory. It is not easy to really motivate the requirements $\mathbf{1 - 2}$, and in fact they simply summarize some ideas of 'naturalness' that are naturally subjective. The first and the second requirement emphasize that in geometry we measure only lengths (we always use the condition that the speed of light $c=1$ ). Requirement 3 is somewhat vague and depends on our understanding of what is 'geometry' and what is 'physics'. Roughly speaking, before we use any Lagrangian we are in the domain of pure geometry. The 'geometric Lagrangian' is a centaur that depends only on geometric variables, but defines a Lagrangian structure through the variational principle and allows to define physical variables.

Clearly, the variables $r_{i j}, s_{i j}, a_{i j}$, and $a_{k} \equiv a_{i k}^{i}$ satisfy requirement 3 . Likewise, we can take as a Lagrangian density Eddington's density (8), which is the simplest possible choice satisfying requirements 1-3. It is easy to see that there exists a wide class of scalar densities also satisfying 13. In the first two papers of [4], Einstein discussed the geometric Lagrangian defined by Eddington's density (8). In the third paper, he assumed that one can take any Lagrangian depending on $s_{i j}$ and $a_{i j}$ as independent variables, i.e.

$$
\mathcal{L}=\mathcal{L}\left(s_{i j}, a_{i j}\right),
$$

but considered a concrete Lagrangian not satisfying requirements 1-2, which in fact is very similar to Weyl's Lagrangian studied in [1]. A simplest generalization of Eddington-Einstein Lagrangian that depends on $s_{i j}$ and $a_{i j}$ and satisfies all the requirements 1-3 was proposed in [9]

$$
\mathcal{L}=\mathcal{L}\left(s_{i j}+\nu a_{i j}\right)=\sqrt{-\operatorname{det}\left(s_{i j}+\nu a_{i j}\right)}
$$

where we take the minus sign because $\operatorname{det}\left(s_{i j}\right)<0$ (due to the local Lorentz invariance requirement) and we naturally assume that the same is true for $\operatorname{det}\left(s_{i j}+\nu a_{i j}\right)$ (to reproduce Einstein's general relativity in the limit $\left.\nu a_{i j} \rightarrow 0\right)$ B

This Lagrangian can be further generalized if we consider other scalar densities of the weight two constructed of $s_{i j}, a_{i j}$, and $a_{i}$. The basic element of the construction is the density

$$
d_{0} \equiv 4 ! \operatorname{det}\left(s_{i j}\right)=\epsilon^{i j k l} s_{i m} s_{j n} s_{k r} s_{l s} \epsilon^{m n r s} \equiv \epsilon \cdot s \cdot s \cdot s \cdot s \cdot \epsilon .
$$

Using the same natural notation we introduce the densities depending also on $a_{i}$ and $a_{i j}$ :

$$
d_{1} \equiv \epsilon \cdot s \cdot s \cdot s \cdot \bar{a} \cdot \epsilon, \quad d_{2} \equiv \epsilon \cdot s \cdot s \cdot a \cdot a \cdot \epsilon, \quad d_{4} \equiv \epsilon \cdot a \cdot a \cdot a \cdot a \cdot \epsilon .
$$

\footnotetext{
${ }^{3}$ In fact, we introduce this new parameter to disentangle the scale of the mass parameter of the vector field from the cosmological constant. It turns out that for $\nu=1$, i.e., for original Eddington - Einstein Lagrangian (8), the mass parameter is close to $\sqrt{\Lambda}$ (see [9]).
} 
where $d_{4}=4 ! \operatorname{det}\left(a_{i j}\right)$ and $\bar{a}$ denotes the matrix $a_{i} a_{j}$. It is easy to find that

$$
\operatorname{det}\left(s_{i j}+\nu a_{i j}\right)=\frac{1}{4 !}\left(d_{0}+6 \nu^{2} d_{2}+\nu^{4} d_{4}\right) .
$$

Then a more general geometric Lagrangian density can be written,

$$
\mathcal{L} \equiv \alpha_{0} \sqrt{\left|d_{0}+\alpha_{1} d_{1}+\alpha_{2} d_{2}+\alpha_{4} d_{4}\right|}
$$

where $\alpha_{i} \in \Re$ and $d_{0}<0$. This Lagrangian is in principle as good as the simplest Lagrangians (8) or (18), although working with it is more difficult and the spectrum of physical models described by it is much wider. Depending on the signs of the numerical coefficients, we could then obtain a positive or a negative cosmological constant and also the standard or the exotic (phantom) sign of the vector field kinetic energy. We note that the Lagrangian has zeroes in the general case, like the simpler Born-Infeld Lagrangian 4

We note that the Lagrangians (8) and (18) are are written in the form independent of $D$, although the analytic expression for the dependence of the determinants on $s_{i j}$ and $a_{i j}$ essentially depends on $D$. In [9], we proposed as one of the requirement a formal independence of the geometric Lagrangian on $D$. Here we omit this requirement because it was poorly formulated and essentially restricts possible Lagrangians to (8) and (18) or, at most, allows to extend $\operatorname{det}\left(s_{i j}+\nu a_{i j}\right)$ to $\operatorname{det}\left(s_{i j}+\nu a_{i j}+\bar{\nu} a_{i} a_{j}\right)$.

The starting point for Einstein (in his first paper of the series [4]) was to write the action principle and to assume that (8) is the Lagrangian density depending on 40 connection functions $\gamma_{j k}^{i}$. Varying the action with respect to these functions, he derived 40 equations that allowed him to find the expression for $\gamma_{j k}^{i}$ given by (14) with $\alpha=-\beta=\frac{1}{6}$.

We reproduced the main steps of the proof in [9]. Here, we somewhat generalize the derivation to an arbitrary dimension $D$ and assume that the geometric Lagrangian depends also on $\gamma_{i} \equiv \gamma_{i m}^{m}$. We define the new tensor densities 5

$$
\frac{\partial \mathcal{L}}{\partial s_{i j}} \equiv \mathbf{g}^{i j}, \quad \frac{\partial \mathcal{L}}{\partial a_{i j}} \equiv \mathbf{f}^{i j}, \quad \frac{\partial \mathcal{L}}{\partial \gamma_{i}} \equiv \mathbf{b}^{i}
$$

and introduce a conjugate Lagrangian density $\mathcal{L}^{*}=\mathcal{L}^{*}\left(\mathbf{g}^{i j}, \mathbf{f}^{i j}, \mathbf{b}^{i}\right)$ by a Legendre transformation,

$$
s_{i j}=\frac{\partial \mathcal{L}^{*}}{\partial \mathbf{g}^{i j}}, \quad a_{i j}=\frac{\partial \mathcal{L}^{*}}{\partial \mathbf{f}^{i j}}, \quad \gamma_{i}=\frac{\partial \mathcal{L}^{*}}{\partial \mathbf{b}^{i}} .
$$

By varying $\mathcal{L}$ in $\gamma_{j k}^{i}$ and using the above definitions, we can then show that the condition $\delta \mathcal{L} / \delta \gamma_{j k}^{i}=$ 0 yields the following 40 equations

$$
2 \nabla_{i}^{\gamma} \mathbf{g}^{j k}=\delta_{i}^{k}\left[\nabla_{m}^{\gamma}\left(\mathbf{g}^{j m}+\mathbf{f}^{j m}\right)-\mathbf{b}^{j}\right]+\delta_{i}^{j}\left[\nabla_{m}^{\gamma}\left(\mathbf{g}^{k m}+\mathbf{f}^{k m}\right)-\mathbf{b}^{k}\right],
$$

where $\nabla_{i}^{\gamma}$ is the covariant derivative with respect to the affine connection $\gamma$. Using the expression for the covariant derivative of the tensor density $\mathbf{f}^{j k}$,

$$
\nabla_{i}^{\gamma} \mathbf{f}^{j k}=\partial_{i} \mathbf{f}^{j k}+\gamma_{i m}^{j} \mathbf{f}^{m k}+\gamma_{i m}^{k} \mathbf{f}^{j m}-\gamma_{i m}^{m} \mathbf{f}^{j k}
$$

\footnotetext{
${ }^{4}$ As a matter of fact, the so called Born-Infeld Lagrangians (see [1] - 15], etc.) are famous descendants of the forgotten Eddington-Einstein Lagrangian.

${ }^{5}$ Following Eddington's notation, we let boldface Latin letters denote tensor densities. The derivatives in (23) and (24) must be properly symmetrized, which is easy in concrete calculations. We tacitly assume that geometry has only a single dimensional constant, e.g., the cosmological constant $\Lambda$ with the dimension $L^{-2}$. Possibly, the characteristic constants for the symmetric and antisymmetric parts differ. To restore the correct dimension in (23) and (24), we must then multiply the densities $\mathbf{g}^{i j}$ and $\mathbf{f}^{i j}$ by $\Lambda_{s}$ and $\Lambda_{a}$. The simplest example of this asymmetry is suggested by Lagrangian (18).
} 
we find that $\nabla_{i}^{\gamma} \mathbf{f}^{k i}=\partial_{i} \mathbf{f}^{k i}$. Then, defining the vector density $\hat{\mathbf{a}}^{k}$ by

$$
\partial_{i} \mathbf{f}^{k i}-\mathbf{b}^{k} \equiv \hat{\mathbf{a}}^{k}
$$

we easily find that

$$
\nabla_{i}^{\gamma} \mathbf{g}^{i k}=-\frac{D+1}{D-1} \hat{\mathbf{a}}^{k}
$$

and hence

$$
\nabla_{i}^{\gamma} \mathbf{g}^{j k}=-\frac{1}{D-1}\left(\delta_{i}^{j} \hat{\mathbf{a}}^{k}+\delta_{i}^{k} \hat{\mathbf{a}}^{j}\right) .
$$

Defining the Riemann metric tensor $g_{i j}$ by the equations

$$
g^{i j} \sqrt{-g}=\mathbf{g}^{i j}, \quad g_{i j} g^{j k}=\delta_{i}^{k},
$$

we can then define the corresponding Riemannian covariant derivative $\nabla_{i}$, for which

$$
\nabla_{i} g_{j k}=0, \quad \nabla_{i} g^{j k}=0 .
$$

Taking the above into account, we can now use (29) to derive the expression for $\gamma_{j k}^{i}$ in terms of the metric tensor $g_{i j}$ and of the vector $\hat{a}^{k} \equiv \hat{\mathbf{a}}^{k} / \sqrt{-g}$,

$$
\gamma_{j k}^{i}=\Gamma_{j k}^{i}[g]+\alpha_{D}\left[\delta_{j}^{i} \hat{a}_{k}+\delta_{k}^{i} \hat{a}_{j}-(D-1) g_{j k} \hat{a}^{i}\right],
$$

which corresponds to $\alpha=\alpha_{D}$ and $\beta=\beta_{D}$ in (14), with

$$
\alpha_{D} \equiv[(D-1)(D-2)]^{-1}, \quad \beta_{D} \equiv-[2(D-1)]^{-1} .
$$

This coincides with Einstein's result for $D=4$ and never gives Weyl's or $g$-Riemannian connection.

We cannot go deeper into discussions of further relations between geometry of affine connections and dynamical principles. But the above results show that these relations are rather complex and we do not yet understand their nature. Having in mind that for different connections the physical models may be drastically different, we tried to add new natural variables into the geometric Lagrangian. However, the class of connections obtained as an output of Einstein's approach did not change at all! It can be argued that there are many other, not yet explored options, but at the moment, we do not even know how to obtain Weyl's or $g$-Riemannian connections following Einstein's approach.

One of the possibilities is to abandon some of Einstein's assumptions. The most serious drawback (or virtue, depending on a viewpoint) of his approach is that two pairs of the basic variables of the theory, $\left(s_{i j}, \mathbf{g}^{i j}\right)$ and $\left(a_{i j}, \mathbf{f}^{i j}\right)$, having very different geometrical and physical meaning, are treated symmetrically. Definition (23) looks quite natural for the metric density because Einstein's Lagrangian for the pure gravity theory is simply $\mathbf{g}^{i j} R_{i j}$. But, Einstein's definition of $\mathbf{f}^{i j}$, in fact, tacitly assumes that the geometric Lagrangian is independent of $\gamma_{i}$ or $a_{i}$ (accordingly, he did not introduce the vector density $\left.\mathbf{b}^{i}\right) 6$ Then it follows that the connection structure is given by (14), where $g_{i j}$ is arbitrary and $\hat{a}_{k}$ is proportional to $a_{i k}^{i}$. Moreover, $\mathbf{f}_{i j} \equiv g_{i m} g_{j n} \mathbf{f}^{m n}$ is proportional to $a_{i j}$. However, in the Einstein approach, the vector $a_{i}$ and the tensor $a_{i j}$ related by Eq.(77) (which exists in the most general geometry) are reproduced with the aid of rather indirect and complex relations (23) and (27).

\footnotetext{
${ }^{6}$ This may look rather paradoxical, but, as we have seen, the mass term is dictated by the geometry, and its trace, the term $\sim a_{i} a_{j}$, is already present in the expression for $s_{i j}$. The physical mass term itself is coming into being when we write an effective physical Lagrangian.
} 
One more question about Einstein's approach concerns the role of the metric tensor $g_{i j}$ in geometry and in physics. In a more general sense, this is a question about the meaning of Einstein's geometric Lagrangian. In Weyl's geometric approach, the tensor $g_{i j}$ is introduced from the very beginning, but it is defined up to the Weyl transformations. In Einstein's approach, it is defined (uniquely, at a first glance) using the Hamilton principle. But we know that the metric depends on the choice of the Weyl gauge (frame) and that the vector field also depends on this choice. In particular, the role of conformal transformations, the choice of the Weyl gauge (frame), and especially the significance and consequences of choosing different independent fields in the initial geometric Lagrangian must be carefully investigated and understood.

\section{Models}

There are many other questions, which should be carefully discussed, but we postpone the discussion to future publications. Here, we present a simple example demonstrating how to eventually pass from geometry to physics. Pure geometry gives us equations (6) and (10). With $a_{j k}^{i}$ given by (14), their right-hand sides are given by $\left(a_{i, j}-a_{j, i}\right) / 2$, where $a_{i}=(D \alpha+2 \beta) \hat{a}_{i}$, and by the sum of (15) and (16) 7 To derive $s_{i j}$ and $a_{i j}$ in terms of the 'physical' variables $g_{i j}$ and $f_{i j}$ we must choose a Lagrangian (e.g., (18)) and then solve equations (23) with respect to the geometric variables $s_{i j}$ and $a_{i j}$. Alternatively, if we know the conjugate Lagrangian $\mathcal{L}^{*}\left(\mathbf{g}^{i j}, \mathbf{f}^{i j}, \ldots\right)$, we can directly calculate them using (24).

In [9], we reproduced Einstein's result (see [4] and [2]):

$$
\mathcal{L} \equiv \sqrt{-\operatorname{det}\left(r_{i j}\right)}=4 \sqrt{-\operatorname{det}\left(\mathbf{g}^{i j}+\mathbf{f}^{i j}\right)} \equiv 4 \sqrt{-\operatorname{det}\left(g_{i j}+f_{i j}\right)}=\mathcal{L}^{*} .
$$

Actually, $\mathcal{L}^{*}=\mathcal{L}$ follows from the fact that $\mathcal{L}$ is a homogeneous function of the degree two, but the concrete expression for $\mathcal{L}^{*}$ must be obtained by a direct calculation. Now we can show that the relation like (34) holds also for Lagrangian (18). For simplicity, we do this by direct calculations in a 'dimensionally reduced' case.

We first define a 'spherical reduction' not using any metric. Suppose that $s_{i j}$ and $a_{i j}$ are functions of $\left(x^{0}, x^{1}\right)$ and that $a_{2}=a_{3}=0$ (therefore, only $a_{01}=-a_{10} \neq 0$ ). We then assume that the symmetric matrix has the following nonzero elements: $s_{i j}=\delta_{i j} s_{i}$, except $s_{01}=s_{10} \neq 0$ (our result will not change if also $s_{23} \neq 0$ ). Explicitly deriving $s_{i j}+\nu a_{i j}$, we can find $\mathbf{g}^{i j}$ and $\mathbf{f}^{i j}$ (using $(\underline{23}))$ and hence derive $\operatorname{det}\left(\mathbf{g}^{i j}+\lambda \mathbf{f}^{i j}\right)$ in terms of $s_{i j}$ and $a_{i j}$

$$
16 \operatorname{det}(\mathbf{g}+\lambda \mathbf{f})=\operatorname{det}\left[s+\left(\nu^{2} \lambda\right) a\right] .
$$

It follows that for $\lambda \nu=1$ we have

$$
\mathcal{L}=-\frac{1}{2} \sqrt{|\operatorname{det}(s+\nu a)|}=-2 \Lambda \sqrt{|\operatorname{det}(\mathbf{g}+\lambda \mathbf{f})|}=\mathcal{L}^{*},
$$

where we introduced the 'cosmological' parameter $\Lambda$ having the dimension $L^{-2}$ (the sign and normalization are arbitrary chosen in relation to the cosmological interpretation). This result is written in the form not implying the spherical reduction, and we suppose it is true in the general four-dimensional theory. In arbitrary dimension $(D \neq 2)$ it must be somewhat modified (see [9]).

We considered the spherical reduction of the four-dimensional manifold. To demonstrate what can be obtained in higher dimension we consider a 'spherically symmetric' five-dimensional model that is dimensionally reduced to dimension four. Let us add to the $4 \times 4$ matrix $r_{i j}=s_{i j}+\nu a_{i j}$

\footnotetext{
${ }^{7}$ These expression are significantly simplified when $\alpha+\beta=0$ (Einstein's connection) or $\alpha-2 \beta=0$ (g-Riemannian connection.
} 
(satisfying all the above requirements) the anti-symmetric elements $a_{i 4}=-a_{4 i}(i=0 . .3)$, which also depend only on $\left(x^{0}, x^{1}\right)$. In addition, we assume that $a_{2}=a_{3}=0$ and that $s_{i 4}=s_{4 i}=s_{4} \delta_{i 4}$, for $i=0, . ., 4$. Then we can immediately calculate $\operatorname{det}\left(s_{i j}+\nu a_{i j}\right)$ :

$$
\prod_{i=0}^{4} s_{i}\left[1-\left(s_{01}^{2}-\nu^{2} a_{01}^{2}\right)\left(s_{0} s_{1}\right)^{-1}+\nu^{2} a_{04}^{2}\left(s_{0} s_{4}\right)^{-1}+a_{14}^{2}\left(s_{1} s_{4}\right)^{-1}-2 \nu^{2} s_{01} a_{04} a_{14}\left(s_{0} s_{1} s_{4}\right)^{-1}\right] .
$$

The terms containing $a_{04}^{2}$ and $a_{14}^{2}$ should be interpreted as kinetic terms of the scalar field $a_{4}$ in four dimensions, because $a_{i 4}=\partial_{i} a_{4} / 2$ while $a_{10}=\left(\partial_{1} a_{0}-\partial_{0} a_{1}\right) / 2$ is the field tensor of the fourdimensional vector field. It can be seen that this scalar field is massive or tachyonic (in the simplest reduction, its mass coincides with that of the vecton).

Taking the square root of the determinant (37) as a geometric Lagrangian, we can construct a two-dimensional model effectively describing spherically symmetric solutions of the four-dimensional gravity coupled to the vecton and scalar fields. By further reductions to static or cosmological solutions we can construct corresponding one-dimensional dynamical systems describing static states with horizons and cosmological models. The cosmological models look realistic enough because they incorporate a natural sources of the dark energy, inflation, and, possibly, some candidates for the dark matter (for a more detailed discussion see [8], [9])). We do not consider a general theory and simply use for $\mathcal{L}^{*}$ expression (36). In the dimension $D$ we must slightly generalize (34) to

$$
\mathcal{L} \equiv \sqrt{-\operatorname{det}\left(s_{i j}+a_{i j}\right)}=\sqrt{-g}\left[-2^{D} \operatorname{det}\left(\delta_{i}^{j}+\lambda f_{i}^{j}\right)\right]^{1 /(D-2)}=\mathcal{L}^{*},
$$

Following the relevant calculations of [9] we can write a 'physical' Lagrangian

$$
\mathcal{L}_{e f f}=\sqrt{-g}\left[-2 \Lambda\left[\operatorname{det}\left(\delta_{i}^{j}+\lambda f_{i}^{j}\right)\right]^{1 /(D-2)}+R(g)+c_{a} g^{i j} a_{i} a_{j}\right],
$$

which should be varied with respect to the metric and the vector field; $c_{a}$ is a parameter depending on $D$ (Einstein's first model is obtained for $D=4$ and $c_{a}=1 / 6$ ). When the vecton field is zero, we have the standard Einstein gravity with the cosmological constant.

The theory described by (39) is very complex, even at the classical level. Its spherically symmetric sector is essentially simpler, but the corresponding two-dimensional field theory is certainly not integrable. We do not know even how to construct its physically interesting approximate solution. Further dimensional reductions to one-dimensional static or cosmological theories also give non-integrable dynamical systems, though some approximate solutions can possibly be derived. A more effective is the small-field approximation formally equivalent to expanding (39) in powers of $\lambda^{2}$. Keeping only the first-order correction we then obtain a nice-looking field theory:

$$
\mathcal{L}_{\text {eff }} \cong \sqrt{-g}\left[R[g]-2 \Lambda-\kappa\left(\frac{1}{2} F_{i j} F^{i j}+\mu^{2} A_{i} A^{i}+g^{i j} \partial_{i} \psi \partial_{j} \psi+m^{2} \psi^{2}\right)\right],
$$

where $A_{i} \sim a_{i}, F_{i j} \sim f_{i j}, \kappa \equiv G / c^{4}$ and we use the CGS dimensions.

This simplified theory still keeps traces of its geometric origin: the simplest form of the dark energy (the cosmological constant $\Lambda$ ), massive (or tachyonic) vector and scalar fields, which can describe inflation and/or imitate dark matter. The most popular inflationary models require a few massive scalar particles usually called inflatons (see, e.g., [16] - 20]). Without massive scalar fields there is no simple inflation mechanism with one massive vecton. However, with the tachyonic vecton (see [21]) or with several massive vector particles, it is probably easier to find a realistic inflation models (see [22] - [27]; some of these papers also discuss possible role of massive vector particles in dark energy and dark matter mechanisms). 
The simplest cosmology can be obtained by the naive reductions using the metric

$$
d s_{4}^{2}=e^{2 \alpha} d r^{2}+e^{2 \beta} d \Omega^{2}(\theta, \phi)-e^{2 \gamma} d t^{2},
$$

where $\alpha, \beta, \gamma$ depend on $t$ and where $d \Omega^{2}(\theta, \phi)$ is the metric on the 2-dimensional sphere $S^{(2)}$. Then the cosmological reduction of four-dimensional theory (40) can be easily found. As was shown in [9], the Lagrangian can be written in the form $\left(A \equiv A_{z}(t)\right)$ :

$$
\mathcal{L}_{c}=e^{2 \beta}\left[e^{-\alpha-\gamma} \dot{A}^{2}-e^{-\alpha+\gamma} \mu^{2} A^{2}-e^{\alpha+\gamma}(V+2 \Lambda)-e^{\alpha-\gamma}\left(2 \dot{\beta}^{2}+4 \dot{\beta} \dot{\alpha}-\dot{\psi}^{2}\right)\right] .
$$

To write the corresponding equations of motion in a most clear and compact form, we introduce the notation

$$
\rho \equiv \frac{1}{3}(\alpha+2 \beta), \quad \sigma \equiv \frac{1}{3}(\beta-\alpha), \quad A_{ \pm}=e^{-2 \rho+4 \sigma}\left(\dot{A}^{2} \pm \mu^{2} e^{2 \gamma} A^{2}\right), \quad \bar{V} \equiv V(\psi)+2 \Lambda .
$$

Then the exact Lagrangian for vecton-scalar cosmology is:

$$
\mathcal{L}_{c}=e^{2 \rho-\gamma}\left(\dot{\psi}^{2}-6 \dot{\rho}^{2}+6 \dot{\sigma}^{2}\right)+e^{3 \rho-\gamma} A_{-}-e^{3 \rho+\gamma} \bar{V}(\psi) .
$$

We see that $A, \psi, \rho, \sigma$ are dynamical variables and $e^{\gamma}$ is a Lagrangian multiplier, whose variations yield the energy constraint:

$$
\dot{\psi}^{2}-6 \dot{\rho}^{2}+6 \dot{\sigma}^{2}+A_{-}+e^{2 \gamma} \bar{V}=0
$$

As in any gauge theory with one constraint of this type, we can choose one gauge fixing condition. The standard conditions are $\gamma=0$ or $\gamma=\alpha$.

The other equations are

$$
\begin{gathered}
\ddot{A}+(\dot{\rho}+4 \dot{\sigma}-\dot{\gamma}) \dot{A}+e^{2 \gamma} \mu^{2} A=0, \\
4 \ddot{\rho}+6 \dot{\rho}^{2}-4 \dot{\rho} \dot{\gamma}-6 \dot{\sigma}^{2}+\frac{1}{3} A_{-}+\dot{\psi}^{2}-e^{2 \gamma} \bar{V}=o, \\
\ddot{\sigma}+3 \dot{\sigma} \dot{\rho}-\dot{\sigma} \dot{\gamma}-\frac{1}{3} A_{-}=0, \\
\ddot{\psi}+(3 \dot{\rho}-\dot{\gamma}) \dot{\psi}+\frac{1}{2} e^{2 \gamma} \bar{V}_{\psi}=0,
\end{gathered}
$$

The system can be simplified by applying a gauge fixing condition. In particular, it can be written in a more standard form by excluding 'dissipative' terms from equations for $\rho, \sigma$, and $\psi$. This can be done by choosing the gauge $\gamma=3 \rho$. In this gauge, the equations of the vecton cosmology are easier to compare with integrable dynamical systems that were extensively studied in cosmology and in black hole theory (see, e.g. [28] - [36]).

Unfortunately, in any gauge these equations remain much more complex than the equations of the scalar cosmology. They are not integrable in any sense and rather difficult for a qualitative analysis. They would be greatly simplified if we could neglect the $\sigma$ field. Unfortunately, this is obviously impossible in general, because then $A_{-}$would be zero and the last condition would be incompatible with the other equations. This means that the exact solutions of the model (even with many scalar fields minimally coupled to gravity) must be non-isotropic 8

In conclusion, we note that the geometrical and dynamical models discussed in this paper are not well understood, both conceptually and technically. Much work on them should be done before a realistic cosmological model could be constructed.

\footnotetext{
${ }^{8}$ If we introduce other scalar fields nonminimally coupled to gravity, then this statement may become not valid. At the moment, we are not ready to add other vector fields or fields with the spin $1 / 2$.
} 
Acknowledgment: It is a great pleasure to dedicate this article to a very old friend, Andrei Slavnov. I am happy to know him for many years and to share his deep vision of science and life. I hope to have many opportunities to see him and to learn from him in future.

This work was supported in part by the Russian Foundation for Basic Research (Grant No. 09-02-12417 ofi-M)

\section{References}

[1] H. Weyl, Raum-Zeit-Materie, Springer, Berlin, 1923 (1-st ed. 1918; English translation 1950).

[2] A.S. Eddington, Proc. Roy. Soc. London A, 99 (1919) 104-122.

[3] A.S. Eddington, The mathematical theory of relativity, Cambbridge Univ. Press, New York, 1923 (German translation of the 2-nd ed. 1925).

[4] A. Einstein, Sitzungsber. Preuss. Akad. Wiss., Phys.-Math., (1923) 32-38, 76-77, 137-140.

[5] A. Einstein, Nature, 112, (1923) 448-449; 'Eddington's Theorie und Hamiltonisches Prinzip', Appendix to the book: A.Eddington, Relativitäts theorie in mathematischer Behandlung, Springer, Berlin, 1925.

[6] E. Schrödinger, Space - time structure, Cambbridge Univ. Press, New York, 1950.

[7] W. Pauli, 'Relativitätstheorie' in: Enzykl. d. Math. Wiss., Vol.5, Teubner, Leipzig (1921), 539-775; Theory of Relativity, Pergamon Press, Cambridge, New York, 1958.

[8] A.T. Filippov, 'On Einstein - Weyl unified model of dark energy and dark matter', arXiv:0812.2616 v2 [gr-qc] (2008).

[9] A.T. Filippov, Theor. Math. Phys. 163(3) (2010) 753-767, arXiv:1003.0782 v2 [hep-th] (2010).

[10] L.P. Eisenhart, Nonriemannian geometry, Amer. Math. Soc. Publ. New York, 1927.

[11] M. Born, Proc. Roy. Soc. London A, 143 (1933/34) 410-437;

M. Born and L. Infeld, Proc. Roy. Soc. London A, 144 (1934) 425-451; 147 (1934) 522-546;

150 (1935) 141-166.

[12] P.A.M. Dirac, Proc. Roy. Soc. London A, 268 (1960) 57-67.

[13] S. Deser and G.W. Gibbons, Class. Quant. Grav. 15 (1998) L35-L39; arXiv:hep-th/9803049v1 (1998).

[14] M. Bañados, Phys. Rev. D, 77 (2008) 123534; arXiv:0801.4103v4 [hep-th] (2008).

[15] D. Langlois, S. Renaux-Petel and D.A. Steer, J. Cosmol. Astropart. Phys., 0904 (2009) 021; arXiv:0902.2941 v1 [hep-th] (2009).

[16] V. Sahni and A. Starobinsky, Internat. J. Mod. Phys. D, 15 (2006) 2105-2132; arXiv:astro-ph/0610026v3 (2006).

[17] A. Linde, 'Particle physics and inflatory cosmology', Harwood, Chur, Switzerland (1990); arXiv:hep-th/0503203v1 (2005).

[18] V. Mukhanov, Physical foundations of cosmology, Cambridge Univ. Press, New York, 2005. 
[19] S. Weinberg, Cosmology, Oxford Univ.Press, Oxford, 2008.

[20] V. Rubakov and D. Gorbunov, Introduction into the theory of early Universe [in Russian], Vols. 1 and 2, Moscow, 2008-2009.

[21] L.H. Ford, Phys. Rev. D, 40 (1989) 967-972.

[22] M.C. Bento, O. Bertolami, P.V. Moniz, J.M. Mourão, and P.M. Sá, Class. Quant. Grav., 10 (1993) 285-298; arXiv:gr-qc/9302034v2 (1993).

[23] C. Armendariz-Picón, J. Cosmol. Astropart. Phys., 0407 (2004) 007.

[24] A. Golovnev, V. Mukhanov and V. Vanchurin, J. Cosmol. Astropart. Phys., 0806 (2008) 009.

[25] T.S. Koivisto and D.F. Mota, 'Anisotropic dark energy: dynamics of backgroud and perturbations', ArXiv:0801.3776v.2 [astro-phys] (2008).

[26] A. Golovnev and V. Vanchurin, Phys. Rev. D 79 (2009) 103524.

[27] C. Germani and A. Kehagias, J. Cosmol. Astropart. Phys., arXiv:0902.3667[astro-phys] (2009).

[28] M. Cavaglià, V. de Alfaro and A.T. Filippov, Internat. J. Mod. Phys. D, 4 (1995) 661-672; 5 (1996) 227-250; 6 (1997) 39-47.

[29] A.T. Filippov, Modern Phys. Lett. A, 11 (1996) 1691-1704; Internat. J. Mod. Phys. A, 12 (1997) 13-22.

[30] H. Nicolai, D. Korotkin and H. Samtleben, 'Integrable classical and quantum gravity', hep-th/9612065 (Cargése lectures).

[31] A. Lukas, B.A. Ovrut and D. Waldram, Phys. Lett. B, 393 (1997) 65.

[32] F. Larsen and F. Wilczek, Phys. Rev. D, 55 (1997) 4591.

[33] H. Lü, S. Mukherji and C.N. Pope, Internat. J. Mod. Phys. A, 14 (1999) 4121.

[34] A.T. Filippov, Theor. Math. Phys., 146 (2006) 95-107; arXiv:hep-th/0505060v2 (2005).

[35] V. de Alfaro and A.T. Filippov, Theor. Math. Phys., 153 (2006) 1709-1731; arXiv:hep-th/0612258v2 (2006).

[36] V. de Alfaro and A.T. Filippov, Theor. Math. Phys., 162 (2010) 34-56; arXiv:0902.4445v.2 [hep-th] (2009). 